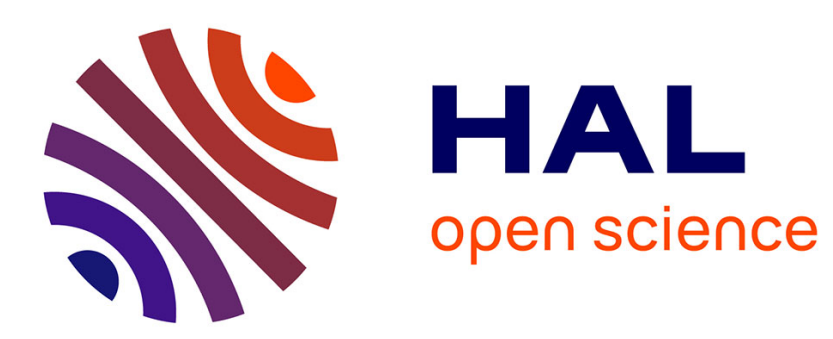

\title{
Experimental and numerical analysis of a novel Darrieus rotor with variable pitch mechanism at low TSR
}

B. Zouzou, I. Dobrev, F. Massouh, R. Dizene

\section{To cite this version:}

B. Zouzou, I. Dobrev, F. Massouh, R. Dizene. Experimental and numerical analysis of a novel Darrieus rotor with variable pitch mechanism at low TSR. Energy, 2019, 186, pp.115832. 10.1016/j.energy.2019.07.162 . hal-02464150

\section{HAL Id: hal-02464150 \\ https://hal.science/hal-02464150}

Submitted on 3 Feb 2020

HAL is a multi-disciplinary open access archive for the deposit and dissemination of scientific research documents, whether they are published or not. The documents may come from teaching and research institutions in France or abroad, or from public or private research centers.
L'archive ouverte pluridisciplinaire HAL, est destinée au dépôt et à la diffusion de documents scientifiques de niveau recherche, publiés ou non, émanant des établissements d'enseignement et de recherche français ou étrangers, des laboratoires publics ou privés. 


\title{
Experimental and numerical analysis of a novel Darrieus rotor with variable pitch mechanism at low TSR
}

\author{
B. Zouzou ${ }^{\text {a, }}{ }^{*}$, I. Dobrev ${ }^{\text {b }}$, F. Massouh ${ }^{\text {b }}$, R. Dizene ${ }^{\text {a }}$ \\ a University of Science and Technology Houari Boumédiène, BP 32 El Alia 16111, Bab Ezzouar, Algeria \\ ${ }^{\mathrm{b}}$ Arts et Métiers-ParisTech, 151, bd L'Hôpital, Paris, 75013, France
}

\begin{abstract}
A B S T R A C T
The Darrieus vertical axis wind-turbine (VAWT) has been the subject of numerous recent studies aimed at improving its aerodynamic performance in order to locate it in urban areas. This article is devoted to the study of an original VAWT with variable-pitch and low tip speed ratio TSR which is favorable to noise reduction and can operate at low velocity wind. The aerodynamic performance of this turbine is studied experimentally in wind tunnel as well as by CFD. In order to obtain the 3D-flow field around the wind turbine rotor, the numerical simulations are performed by means of detached eddy simulation method (DES). The simulation of pitch variation is made possible by using sliding-mesh method. Thus a specially created program adapts the pitch depending on the blade azimuthal position during rotation. The obtained results show that adapted pitch blades are preferable because they permit to obtain a power coefficient $\mathrm{Cp}$ that rivals other VAWT in the case of $T S R<1$. The maximum torque fluctuation during rotation is lower in the case of adapted variable-pitch compared to fixed-pitch and thus the maximum aerodynamic loads on the structure can be reduced. Moreover, the pitch adaptation leads to lower interaction effects between the upstream-blade wake and down-stream blades.
\end{abstract}

\section{Introduction}

Wind energy is considered one of the most promising energy resources because of its cleanliness (no air pollution) and easy access. As a typical devices that converts wind energy into electricity, wind turbines have attracted a lot of interest in recent years [1]. Wind turbines can be classified into two main types according to their axis of rotation: horizontal axis wind turbines (HAWT) and vertical axis wind turbines (VAWT). Wind turbines are often installed in open rural areas. However, in recent years, there have been growing interest in the use of these turbines in urban areas. The main objective is to produce energy on site, thus reducing energy transport costs and losses [2]. HAWTs have long been used in large wind farms because they are known to be more efficient than VAWTs under stable and constant wind conditions. Smallscale HAWTs are also increasingly used in urban areas. However, various studies have shown that VAWTs perform better in urban areas than HAWTs [2,3]. These advantages are mainly due to various reasons, the most important of which is VAWT's ability to

\footnotetext{
* Corresponding author.

E-mail address: bzouzou@usthb.dz (B. Zouzou).
}

operate in a multidirectional wind that is likely to change permanently in residential areas. Unlike HAWTs, VAWTs do not require a yaw control mechanism and react rapidly to changes in wind speed and direction, making them more effective in turbulent regions and also giving them the ability to be quieter. According to aerodynamic principles, VAWTs can be classified into drag and lift turbines. The Darrieus wind turbine is a lift turbine. This type of wind turbine was designed by Jean Marie Darrieus in 1931 [4]. The Darrieus rotor blades can be curved or helical, and can be fixed-pitch or controlled by a variable-pitch system. Darrieus VAWTs with fixed-pitch blades have some disadvantages, such as difficult self starting, low efficiency and reduced service life due to high fluctuations [5], unlike wind turbines, original variable pitch wind turbines with different mechanisms, which describe a cycloidal motion among them the Voith-Schneider mechanism [6] and VTOL (vertical take-off and landing aircraft) [7]. There are several aspects in which a variablepitch design can improve conventional VAWT and HAWT machines (Reference [8] for more details). In variable-pitch turbines, the angle of attack can be adjusted by modifying it. By controlling the angle of attack, variable pitch turbines can significantly increase the starting torque, resulting in the self start of a turbine. In addition, a Darrieus VAWT with variable pitch can generate high efficiency and 
reduce torque ripple [9]. Fig. 5 shows the schematics of an original VAWT mechanism with variable pitch adapted by ADVTECH [10]. This design is a mechanism that is based on the LIPP-Mvt, the angle between the chord line and the tangent to the trajectory depends on the eccentricity between the axis of rotation and the cam. In this paper, the characteristics of the variable-pitch VAWTs are discussed. Our study, is based on the advantage of high-solidity wind turbines and combines them with the advantages of variable pitch wind turbines. The researcher's efforts focused mainly on improving and eliminating the negative effects of a dynamic stall. The complex flow phenomena associated with the Darrieus fixed and original variable pitch wind turbine make it difficult to predict its performance, which will be an interesting work to explore. Since a detailed observation of the flow structure is required in this study, detailed computational fluid dynamics (CFD) calculations are used to correctly predict the flow behaviour around the rotor. This is due to the complexity of VAWT's flow structures. To overcome the complex flow phenomena associated with the Darrieus wind turbine, most of the studies reviewed above were all limited to 2D simulations using 2D-flow models, and many of them were conducted on a specific TSR. HAWTs normally achieve a power coefficient value of $C_{P}=0.4$ to $C_{P}=0.45$ [11]. In variable-pitch VAWTs, $C_{P}$ values between 0.35 and 0.4 were obtained 20 years ago [12] see Fig. 1. These studies did not investigate the effects of a high-solidity variable pitch system with LIPP-Mvt to improve turbine performance. In addition, flow on turbines with high-solidity and low TSR deviates from 2D behaviour when the effects of flow separation become significant, making flow three-dimensional. In order to overcome these limitations, this work capitalizes on these aspects and aims to build a credible 3D CFD model. In this context, the main objective of this paper is to study the aerodynamics of a 3D flow around a VAWT with a high fixed and variable pitch solidity using a UDF (user-defined function) that combines two movements: the azimuth rotation of the blades and the variation of the pitch angle. The DES turbulence model (Detached eddy simulation) was chosen in order to obtain precise results and reduce calculation costs. The sliding mesh was adopted to generate the mesh topology. To demonstrate the advantages of the original variable pitch wind turbine with a high-solidity compared to the fixed pitch wind turbine with a high-solidity. This article deals specifically with the power and torque coefficients of the studied wind turbines, and the distribution of rotor vortex structures and the innovative variable pitch blade mechanism based on LIPP movement. The DES model is

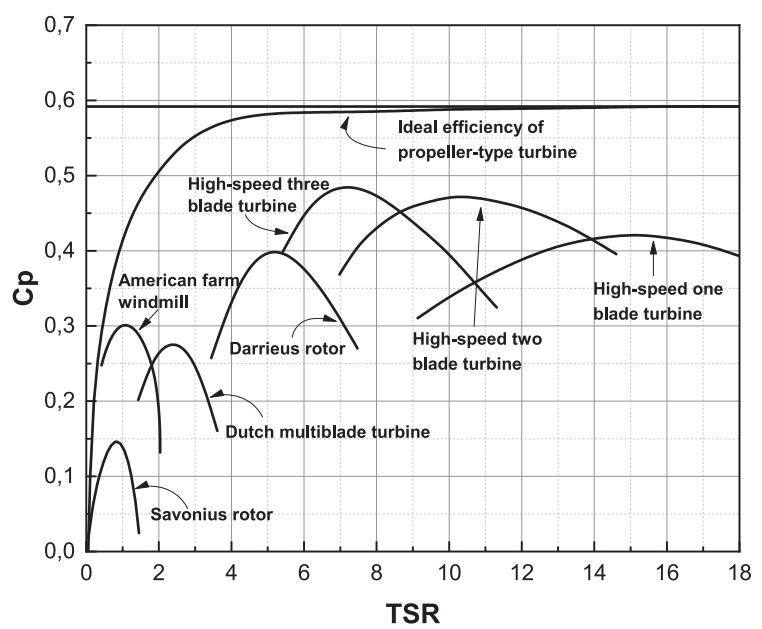

Fig. 1. Typical evolution of the power coefficient as a function of the TSR and the wind turbine model. used with a 3D modelling approach due to the complexity of the flow and the considerable wake between the three blades. In addition, we are examining VAWT's aerodynamic capacity with variable blades, in particular its ability to reduce dynamic stall at relatively low TSRs and their ability to facilitate their dissipation. It should be noted that any future attempt to improve this new design of VAWT technology with high variable pitch solidity should be facilitated by the use of 3D simulations.

\section{Review analysis}

Paraschivoiu et al. [13] analyzed an H-Darrieus prototype turbine with a pitch control system. In this study, the CARDAAV code was developed on the basis of the "Double-Multiple Streamtube" model to determine the performance of VAWT straight blade. The code was coupled employing a genetic algorithmic program optimizer and azimuthal variation of blade pitch angle was modeled using an analytical function with variable coefficients of the improvement method. In the variable angle methodology, the blade angle of attack changes according to local flow conditions. An increase of nearly $30 \%$ in annual energy production is obtained by using polynomial control of the optimum pitch amplitude [13]. Bianchini et al. [14] have optimized the pitch system of a small Darrieus wind turbine. The utilization of a BEM code is advancing so as to model the variable pitch to check different blade pitch management methods. The results showed that with an appropriate pitch angle, the turbine output power could increase considerably. Li et al. [15] used computational fluid dynamics to investigate the unsteady simulation of two-dimensional flow. Simulations show that optimized pitch amplitude curves will increase the average power coefficient for various tip speed ratios (TSRs). Rezaeiha et al. [16] used Unsteady random averaged Navier Stokes (URANS) and SST turbulence model to study the performance of a Darrieus turbine for various inclination angles. The results showed that a $6.6 \%$ increase in the power coefficient can be achieved by employing $\mathrm{a}-2^{\circ}$ tilt angle at a peak speed ratio of four. Jain and Abhishek [17] developed an aerodynamic model based on the Double Multiple Stream Tube for a small scale VAWT. Additionally, unsteady aerodynamic effects were modeled using the Wagner function. The authors showed that by decreasing the amplitude of the change in pitch amplitude from $30^{\circ}$ to $8^{\circ}$, a peak power coefficient greater than 0.3 can be achieved and maintained between peak speed ratios of 1-2.5. Abdalrahman et al. [18] studied the effects of fixed and variable pitch blades on the performance of a Darrieus turbine using CFD simulation. A 2D simulation of unsteady flow with a moving mesh and $k-\omega$ turbulence model. The results showed that a blade pitch control system can be used for a H-VAWT to improve its energy production performance. The authors also found that the variable angle blade increased torque and starting efficiency while reducing torque fluctuations. Sagharichi et al. [9] used CFD and studied the effects of pitch angle for fixed and tangent blades to improve the performance of a VAWT, and proposed a pitch system to reduce the oscillatory motion of the blades and control the attack during rotation. Their numerical results showed that the turbulence model SST $k-\omega$ corresponds well with the experimental results. Xisto et al. [19] studied four key parameters that strongly influence the performance of a VAWT and original variable pitch wind turbine, using a 2D CFD with URANS turbulence model validated from experimental data. Kavade and Ghanegaonkar [20] studied the best pitch position of each blade in the turbine rotation at different azimuth angles for different TSR. The results indicate that the optimized blade pitch with pitch variations from $45^{\circ}$ to $10^{\circ}$ improves turbine performance for a TSR of $0<\lambda<3$. Shahizare et al. [21] studied the output 
power of VAWTs in urban areas by examining the effects of the omnidirectional angle. Gharali and Johnso [22] and Almohammadi et al. [23] studied the 2D dynamic stall of a blade and a complete straight blade respectively, and Gharali stated that a threedimensional (3D) model was a better choice for studying the dynamic stall phenomenon. Lei et al. [24] performed an unsteady CFD numerical analysis on the performance of an offshore VAWT floating in different amplitudes and pitch periods. The results showed that pitch motion can increase the range of variation of aerodynamic force coefficients. Lee and Lim [25] studied various parameters that affect VAWT performance, including rotor diameter, blade length, pitch angle and helical angle. The results guided the design of the technology. In order to improve the initial self starting torque, the use of high-solidity rotors is preferable. The effects of solidity on the performance of a fixed-pitch wind turbine have been the subject of numerous studies. Mainly Roh and Kang [26] who studied different rotor solidities ranging from 0.5 to 0.8 . It is indicated that by increasing solidity, the power curve's evolution of the Darrieus VAWT straight type varies from that of the Darrieus VAWT Eggbeater type. More recently, Kirke and Paillard [27] have used two different models (DMS and RANS 2-D) to predict the performance of the vertical axial turbine in fixed and variable pitch. They stated that the variable pitch turbine can overcome two major disadvantages, the inability to start itself and the overspeed of fixed pitch vertical axis wind turbines. Overall, it could be argued that vertical axis wind turbines, and among them mainly Darrieus wind turbines, are not yet fully developed. The disadvantage of highsolidity wind turbines is a low power coefficient. Unlike the original variable pitch wind turbine, who solves this problem.

\section{Experimental system}

The wind turbine shown in Fig. 2 is designed and patented by the company ADV TECH. It is a vertical axis wind turbine whose blades have a variable pitch during rotation. In order to determine the operating characteristics of the wind turbine and to improve its performance, experiments in the wind tunnel under controlled wind conditions were carried out. Thus, several measurement campaigns were conducted to determine the wind turbine's performance at different variable pitch laws. This wind turbine has 3 straight blades with a specific mechanism that allows the blade angle to vary according to the rotor's azimuth position. This variable pitch setting of the blades will ensure their orientation respectively to the wind with an "optimal" incidence below the stall angle. Thus, the wind turbine's aerodynamic efficiency is improved. Unfortunately due to the dynamic stall phenomenon, the "optimum" pitch variations ratio cannot be calculated in advance, even with powerful simulation tools. Thus wind tunnel tests are necessary.

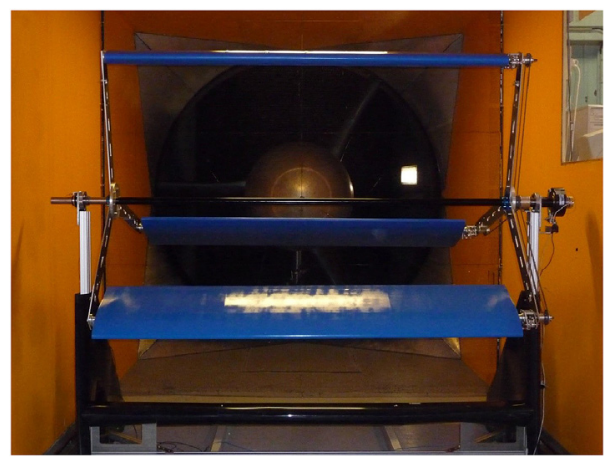

Fig. 2. ADV TECH prototype tested in wind tunnel.

\subsection{Test bench(wind tunnel)}

The ENSAM wind tunnel is a closed return wind tunnel (Prandtl type) with semi-guided open test section with cross section dimensions of $1.35 \mathrm{~m} \times 1.65 \mathrm{~m}$ and $2 \mathrm{~m}$ of length (Fig. 3). The wind tunnel fan is equipped with variable-frequency drive and the wind velocity in the test section can vary from 1.5 to $40 \mathrm{~m} / \mathrm{s}$. The test section is preceded by a settling chamber, equipped by honeycomb panels and a nozzle with contraction ratio of 12.5. These conditions permit to insure good uniformity of velocity in the test section and to obtain a turbulence intensity lower than 5 thousandths. The velocity in the test section is calculated using Bernoulli equation, assuming that the velocity in the settling chamber is quite low and can be neglected:

$V_{\text {ref }}=\alpha \varepsilon \sqrt{\frac{2 \Delta p}{\rho}}$

In this equation the flow coefficient $\alpha \varepsilon$ is very close to 1 . The pressure drop $\Delta p$ between the test section and settling chamber is measured by a precision micro manometer Furness Controls FCO510 with two ranges: $0-200 P a$ and $0-2000 P a$. The accuracy specified by the manufacturer is $0.1 \%$ between $10 \%$ and $100 \%$ of reading scale. The air density is calculated from the atmospheric pressure which is measured by mercury barometer and the air temperature which is measured by PT100 Class $1 / 3$ DIN. Due to dimension limitation of the test section, the wind turbine is installed at the return channel of the wind tunnel which has $3 m \times$ $3 m$ section and a length of $6 m$. This return channel is managed to allow the study of horizontal or transverse axis wind turbines. Viewing the ratio (4.0404) between return channel and test sections, the mean velocity $\mathrm{V}$ in the return channel is:

$V=0,2475 \sqrt{\frac{2 \Delta p}{\rho}}$

\subsection{Wind turbine instrumentation}

For the calculation of the wind turbine power P, the ADV Tech wind turbine is instrumented with a torque measurement system and an angular velocity measurement system. The torque measuring system includes, Fig. 4, an electromagnetic powder brake Merobel FBB which is installed on the wind turbine shaft. The

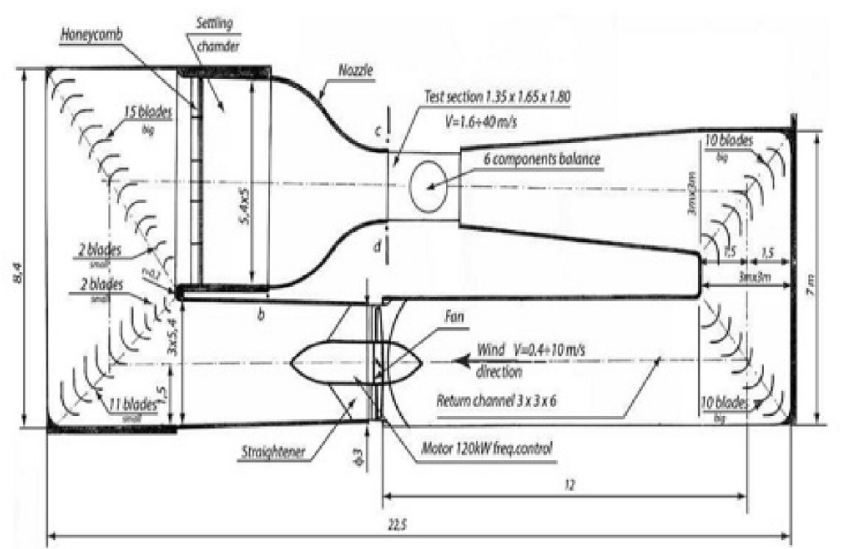

Fig. 3. 


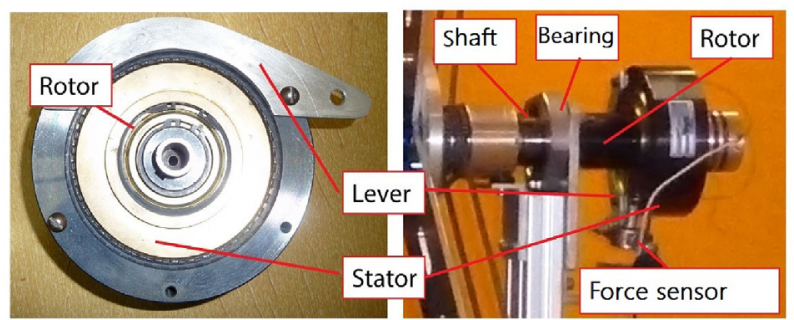

Fig. 4. Braque and torque meter.

torque of brake depends on the intensity of the electrical current supplying the brake, which is controlled by the computer. Thus, during the tests, the variation in current intensity allows to study the wind turbine under different load conditions. The stator of the brake is attached to the nacelle by means of rigid lever and strain gauge load cell SEDEME type AC 50 daN of accuracy class 0.2 . Thus, the torque $T$ exerted by the brake is related to the force measured by the load cell. The cell is connected with HBM MGCplus data acquisition system equipped with measurement card ML30B of accuracy class 0.03 . The measured torque includes both the rotor aerodynamic torque, the torque due to aerodynamic drag of blade support struts and mechanical loses of variable pitch system. The losses in variable pitch system are quite low as the blade pitching axis is close to the aerodynamic center of the blade section. It should be noted that aerodynamic and mechanical power loses of this wind turbine are quiet low compared to other lift type VAWT. This is due to the fact that its angular velocity is lower than others VAWT when operating at the same wind. The angular velocity measuring system includes a disc with holes, installed on the shaft of the wind turbine, and an optical sensor Keyence FS-V21 which is pointing the holes. The number of pulses emitted by the sensor during a given time allows calculating the angular velocity $\omega$ of the wind turbine. By measuring the torque and angular velocity, the power $\mathrm{P}$ of the wind turbine can be calculated as:

$P=T \omega$

\section{Procedure applied for the tests}

The tests are conducted at air velocity $\mathrm{V}$ of $5 \mathrm{~m} / \mathrm{s}$. For this velocity, the variable frequency drive which controls the wind tunnel ventilator permits to obtain a standard deviation less than $0.015 \mathrm{~m} / \mathrm{s}$ computed over a time interval of $180 \mathrm{~s}$. A computer with LabVIEW program is connected to HBM MGCplus data acquisitions system which measures torque $\mathrm{T}$, pressure in the settling chamber $\Delta p$ and air temperature t. The computer is equipped with NI PCle6259 card which permits measurement of angular velocity and enables changing current intensity applied to the brake. The sampling rate and measurement time are defined by the user, so that the average values of the signals can be obtained correctly. Generally, a measurement time of 180 s is sufficient for a sampling rate of $4 \mathrm{~Hz}$. The integration time for angular velocity measurement is set to $2 s$. At the end of each measurement series for a given operating point, the results are saved in a file so that they can be evaluated and analyzed. Nevertheless, in order to facilitate wind turbine control, the software displays the instantaneous values of wind speed $\mathrm{V}$, air temperature $\mathrm{t}$, rotational speed $\omega$, torque $\mathrm{T}$, power coefficient $C_{p}$ and tip speed ratio $\lambda$. The analysis of values of measured variables and the accuracy of the measuring system give an uncertainty of power coefficient less than $3.5 \%$ and for specific speed less than $1.5 \%$.

\section{Variable pitch mechanism system}

The specificity of the studied wind turbine is the rotation of its blades according to the azimuth angle, the variation mechanism of which is described in a patent [10]. The leading edge of the blade is always facing upwind (Fig. 7, Fig. 5b). By convention, the blade pitch angle is measured with respect to the horizontal axis. For an azimuth angle of $0^{\circ}$ or $360^{\circ}$ the blade pitch angle is in the rear position. This angle is always positive. On the other hand, for an azimuth angle of $180^{\circ}$, the blade pitch angle is positioned in front of the blade. As a result, it is always negative. Note that in the $90^{\circ}$ and $270^{\circ}$ azimuth positions, the setting angle is equal to $0^{\circ}$ and the blade is horizontal Fig. 5 .

$$
\begin{aligned}
& \alpha_{0}=\tan ^{-1}\left(\frac{\sin \theta}{\lambda+\cos \theta}\right) \\
& \alpha=\tan ^{-1}\left(\frac{\sin \theta}{\lambda+\cos \theta}\right)-\varphi
\end{aligned}
$$

Where the relationship between angle of attack (AOA) and the azimuth angle can be plotted in Fig. 6, which shows the difference

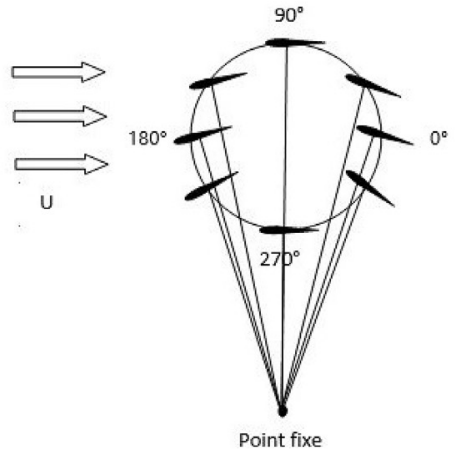

(a) Example of trochoidal trajectory for a

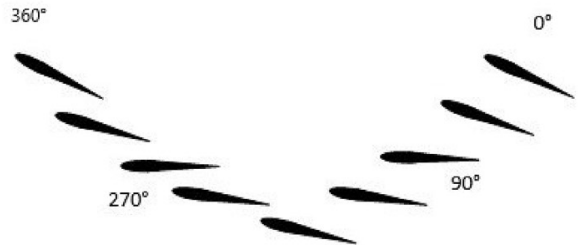

(b) Example of trochoidal trajectory 


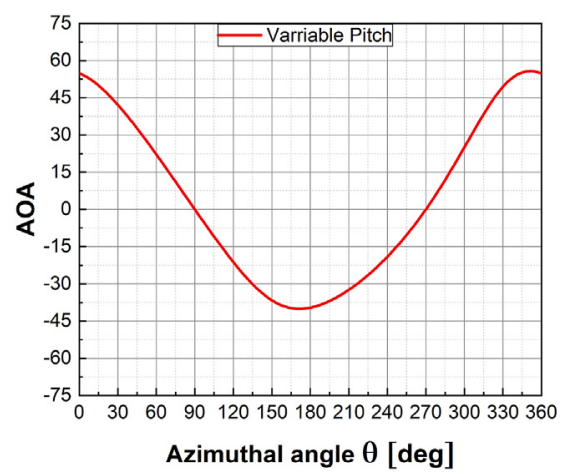

Fig. 6. Variation of blade angle of attack during one revolution for different cases.

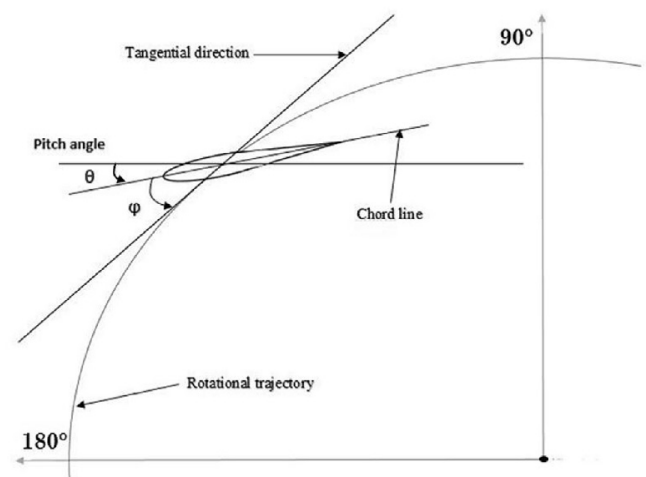

Fig. 7. Schematic representation of variable pitch blade.

between the variable pitch laws. These two laws were chosen for their $\mathrm{Cp}$ max performance in the experimental study. Adjusting the pitch angle could reduce the AOA values for the upstream airfoil profile, thus reducing the dynamic stall range. In fact, the AOA of the downstream blades are smaller than those shown in Fig. 6, since the downstream induced speed is lower than the speed of the free current. It also reduces downstream dynamic stall. On the other hand, this original variable pitch wind turbine has considerably reduced the dynamic stall for $\lambda<1$. As for symmetrical blades, Sheldahl and Klimas [28] pointed out that the dynamic stall often occurred at AOA values ranging from $10^{\circ}$ to $15^{\circ}$, and dynamic stall always occurred at (TSR) $\lambda<4$. Therefore, the large AOA is a sufficient condition of dynamic stall, and it is necessary to study the aerodynamic forces of the blades when operating at a lower TSR. From Fig. 6, the maximum angle of attack of a blade profile can reach about $54^{\circ}$.

\section{Computational and numerical approach}

\subsection{DES turbulance model}

The approach to modelling turbulence should be as uncomplicated as possible while providing an accurate representation of the physics of dynamic stall. The simulation of large eddies (LES) is a powerful turbulence model that has attracted more attention in recent VAWT studies. The 3D model LES was used by Elkhoury et al. [29], resulting in a good agreement with the experiments. Although the LES method is considered a very fine method, it requires an enormous calculation cost. In order to obtain precise results and reduce calculation costs, a method combining the URANS and LES models has been suggested, one of which is the (DES based on 2 equations). In the feasible DES method, small near-limit eddies were modeled using an URANS formula and large far-field eddies were simulated using an LES model. The DES is a non-zonal approach and provides a single smooth velocity field in the RANS and LES regions of the solution. This RANS model is similar to the Realizable $k-\varepsilon$ model, with the exception of the dissipation term in the $k$ equation. In the DES model, the Realizable $k-\varepsilon$ RANS dissipation term is modified such that:

$Y_{k}=\frac{\rho k^{\frac{3}{2}}}{l_{d e s}}$

where

$$
\begin{aligned}
& l_{\text {des }}=\min \left(l_{r K e}, l_{\text {les }}\right) \\
& l_{\text {des }}=\min \left(l_{r K e}, l_{l e s}\right)
\end{aligned}
$$

For the case where $l_{\text {des }}=l_{\text {rke }}$, you will obtain an expression for the dissipation of the $k$ formulation for the Realizable $k-\varepsilon$ model.

\subsection{Numerical method}

Generally speaking, the phenomenon can be considered incompressible when the Mach number is less than 0.3 [30], its value is generally very low in the context of small wind turbines, about $10^{-2}$ to $10^{-1}$. As the Mach number of the flow for our VAWT variable blade wind turbine is at about 0.007 then we'll use the incompressible 3D Navier-Stokes equations as resolution equations. The DES model acts as a turbulence model. Ansys fluent is used as solver with an UDF to interpret the motion of the wind turbine VAWT with variable pitch [31]. The Ansys fluent software is based on the finite volume method, it is used to solve the N-S equations.

\subsection{Geometry}

The 3D geometrical model of the VAWT is shown in Fig. 8 The aerodynamic blade profile is NACA 0018 with a chord length of $c=$ $0.04 \mathrm{~m}$ and a rotor diameter of $D=1 \mathrm{~m}$ (Fig. 9a) with a blade span $H=2 \mathrm{~m}$ (Fig. 9b). The pitch angle of the blade (as shown in Fig. 7) is adjusted according to LIPP's motion based on variable pitch laws as shown in Fig. 6 according to the experimental parameters. Solidity is defined as $N c / D=1.2$ for our case, where $\mathrm{N}, \mathrm{c}$ and $\mathrm{D}$ are the number of blades, the length of the chord and the diameter of the rotor respectively Table 1 .

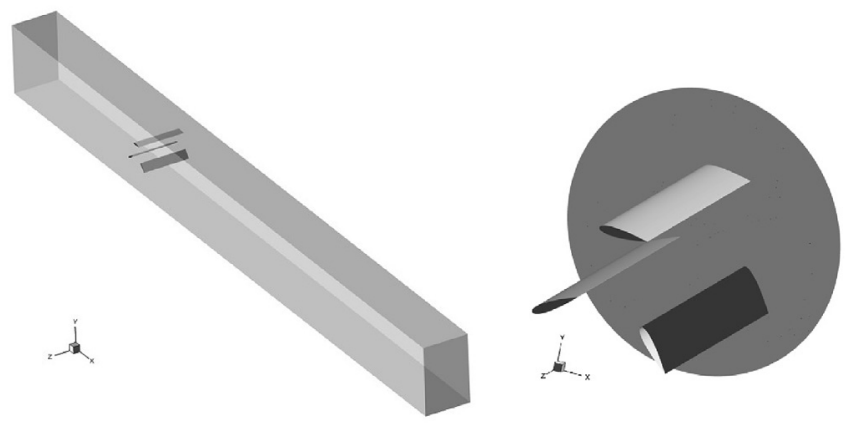

Fig. 8. A 3D overview of the modeled configuration. 


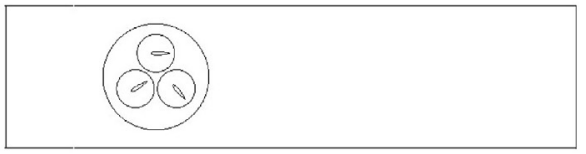

(a) lateral view

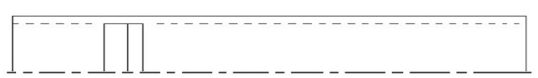

(b) top view

Fig. 9. Computational domain.

Table 1

Darrieus wind turbine specifications.

\begin{tabular}{ll}
\hline Parameter & Value \\
\hline Chord, c & $0.4[\mathrm{~m}]$ \\
Solidity, $\sigma$ & 1.2 \\
Number of blades, $\mathrm{n}$ & 3 \\
Blade span, H & $2[\mathrm{~m}]$ \\
Diameter, d & $1[\mathrm{~m}]$ \\
Airfoil & $\mathrm{NACA} \mathrm{0018}$ \\
Freestream velocity, $U_{\infty}$ & $5[\mathrm{~m} / \mathrm{s}]$ \\
\hline
\end{tabular}

\subsection{Boundary conditions}

In order to simulate the performance of the VAWT, an appropriate 3D computation domain is created. A 2D diagram is shown in Fig. 9 for more details. The computational domain contains five parts: three rotating zones that contain the blade, another one that is the rotor and a static zone, this domain has a 3D of width and a length of 16D [32]. The height of the computational domain corresponds to the geometrical characteristics of the wind tunnel. The entire 3D domain is illustrated in Fig. 8.

A sliding mesh technique is used to simulate rotor rotation and blade pitch characteristics. The contact surfaces between the rotating and stationary zones are defined as the interface, on which the zones can exchange data after each time step. The mesh size of the two areas in the interface should be consistently generated as far as possible, so that the efficiency and accuracy of the data exchange can be guaranteed. The boundary conditions are shown in Fig. 10. In order to simulate the experimental conditions and compare the numerical results with experimental data, interfaces are defined on the contact surfaces of the rotating and static zones. The blade surface, inlet and outlet are defined as the, velocity inlet $(5 \mathrm{~m} / \mathrm{s})$ and pressure outlet respectively. A symmetry condition for the left surface, while for the upper, lower and right wall surfaces of the fixed area, the condition is defined as a non-slip wall.

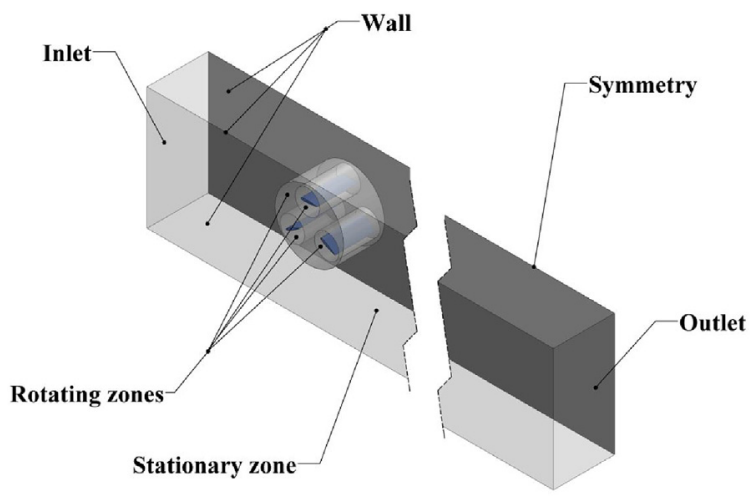

Fig. 10. Boundary conditions specification.

\subsection{The meshing}

The aim of this work is to study the wind turbine performance and aerodynamic characteristics of the blades. Therefore, the mesh size around the blades should be sufficiently defined. At the same time, prismatic boundary layer cells must be provided on the blade surface. The total thickness of the boundary layer is $0.025 \mathrm{~m}$ with a growth rate of 1.2, as shown in Fig. 11. The mesh topology in 3D is illustrated at Fig. 12, the input flow velocity is $5 \mathrm{~m} / \mathrm{s}$, and the Reynolds number based on the length of the chord $(0.4 \mathrm{~m})$ is $R e=1.3 \times$ $10^{5}$. Therefore, the height of the first prismatic cells is set at $0.00003 \mathrm{~m}$ which satisfies the requirements of the DES model for mesh resolution. The TSR can be defined as $\lambda=\omega r / v$, where $\omega r$ is the angular speed of the rotor and $v$ is the speed of the infinite upstream, in our case we have taken a mesh with about 19.53 million cells in order to better reflect the complex motion.

\subsection{The resolution}

A method is chosen to solve discretized equations of continuity and moment, and the second order formula is used for discrete differential equations. The SIMPLE algorithm is used to solve the pressure-speed coupling equation while the DES turbulence model is used to solve the turbulent viscosity term. The incompressible equations of Navier-Stokes can be summarized by:

$\frac{\partial U_{i}}{\partial X_{i}}=0$

$\frac{\partial \bar{U}_{i}}{\partial t}+\frac{\partial}{\partial X_{i}}\left(\bar{U}_{i} \bar{U}_{j}\right)=-\frac{1}{\rho} \frac{\partial \bar{P}}{\partial X_{i}}+v \quad \nabla^{2} \bar{U}_{i}-\frac{\partial \tau_{\mathrm{ij}}^{S}}{\partial X_{j}}$

The convergence criterion is considered satisfied when the moment coefficient becomes cyclical and stable. To improve computing efficiency, 7 parallel CPU kernels are selected to solve equations. It takes about $210 \mathrm{~h}$ to reach six calculation turns. In instationary calculations, setting the time step is a very critical problem [33]. There have been many studies on the effect of time on computation results. Elkhoury et al. [29] studied a 3-blade VAWT, the time step was set to the values corresponding to the time during which the rotor rotated $2 p / 300$ and $2 p / 600 \mathrm{rad}$, respectively, and they found that both time steps had almost no effect on the instantaneous torque.
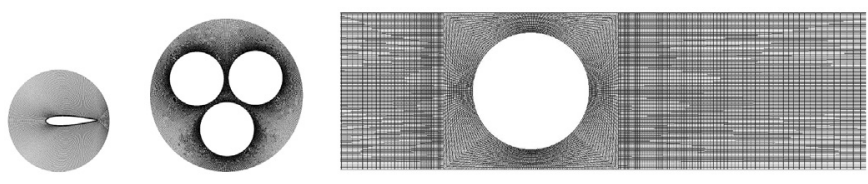

Fig. 11. Computational domain grid details front view. 

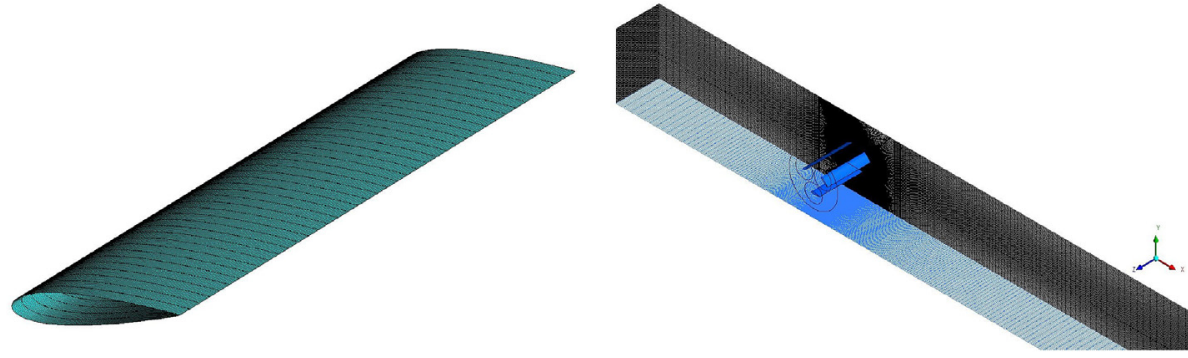

Fig. 12. Computational domain grid 3D details.

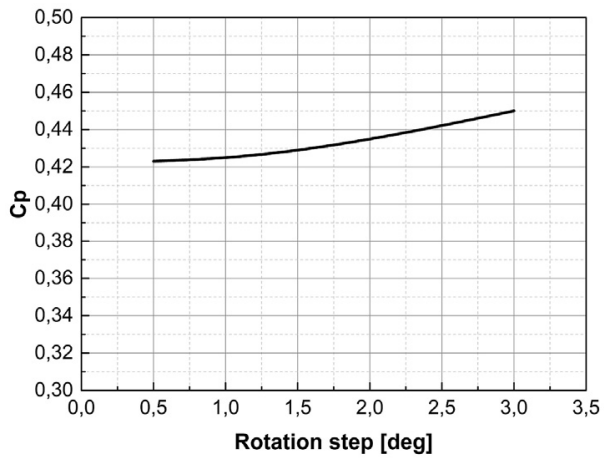

Fig. 13. Effect of time step on solution accuracy at $\mathrm{TSR}=0.52$.

In our case, we tested 3 time steps equivalent to degrees of rotation: each $0.5^{\circ}, 1^{\circ}, 3^{\circ}$. It can be seen that there is practically no difference between the steps of $0.5^{\circ}$ and $1^{\circ}$ (Fig. 13). As a result, the time step is set on a step equivalent to $1^{\circ}$ in this study in order to reduce the calculation time. In order to obtain an accurate aerodynamic force and information on the stable velocity field, the maximum physical time must be long enough so that information on the stable flow field can be obtained after several rotor rotations. Fig. 14 shows the instantaneous values of torque changing over time, which shows that the adjacent peaks of torque have practically no change (error less than 1\%) after 7 to 8 rotations of the rotor (Fig. 15). Therefore, the performance of a VAWT and the speed field is considered to be stabilized after 8 rotations, indicating that the maximum physical time is equal to 7 rotor rotation periods.

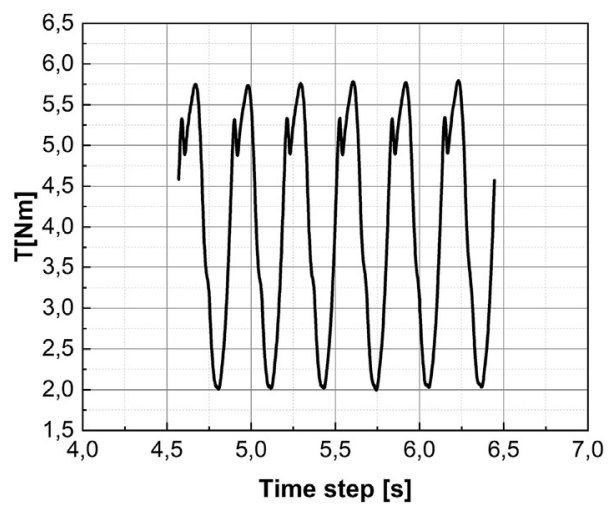

Fig. 14. Torque values as a function of time during a rotation for the original variable pitch wind turbine.

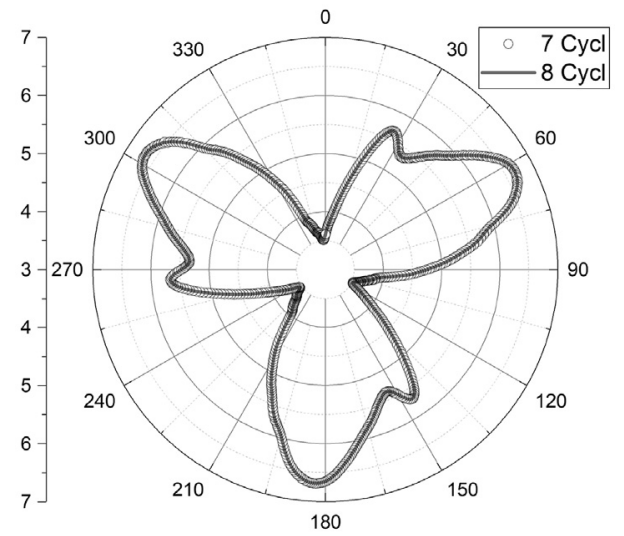

Fig. 15. Torque values for the original variable pitch wind turbine with different cycles.

\section{Results and discussions}

The experimental results conducted on the original variable pitch wind turbine were performed by including mechanical losses. The numerical simulations were carried out for two configurations: a fixed pitch wind turbine and an original variable pitch wind turbine. The blade angle of this wind turbine was converted to numerical from the experimental, modelling the complex azimuthal rotation motion and blade pitch, were used in ANSYS Fluent using a user defined function (UDF). This numerical study is based on the blades without the axis of rotation nor the linkage bars. It should be noted that solidity plays a major role in the TSR at which a wind turbine achieves its maximum power coefficient. Low solidity VAWT reaching their maximum efficiency at a TSR between 3 and 5 showed maximum power coefficients of less than 0.4 . Given the high-solidity of these conventional VAWT $\sigma=1.2$, its maximum $\mathrm{Cp}$ values are expected to be even lower for low solidity wind turbines, 0.2 for Savonius wind turbine. Low solidity wind turbines are typically used for low torque and high speed. While high-solidity wind turbines are used for high torque, low speed. The latter scenario is of interest for the present design as relatively low speeds reduce noise and vibration due to imbalance of wind turbine rotor. This will maximize turbine life while producing acceptable power. In the following paragraphs, the effects of power coefficients, pressure, and turbulent intensity on VAWT performance are assessed. It is worth mentioning that 5 different TSR cases were numerically simulated for each of the tests considered because the calculation cost was substantially excessive.

\subsection{The effect of power coefficient}

One of the important parameters for a wind turbine is the Power 
coefficient (Cp), which is a measure of turbine efficiency and is defined as [34].

$P_{\text {wind }}=\frac{1}{2} \rho A V^{3} C_{p}$

$C_{p}=\frac{P_{T}}{P_{\text {wind }}}, C_{p}=\frac{P_{T}}{\frac{1}{2} \rho V^{3} A}$

where $\rho\left(\mathrm{kg} / \mathrm{m}^{3}\right)$ and the specific mass of air, $V(\mathrm{~m} / \mathrm{s})$ wind speed to the wind turbine's infinity upstream, $A\left(\mathrm{~m}^{2}\right)$ surface swept by the rotor. The power coefficients are compared in Fig. 16. Only the trend of variation in the power coefficients predicted by the two settings can be seen. In Fig. 16, the curve shows the evolution of the power coefficient of the $\mathrm{Cp}$ wind turbine, as a function of the specific speed of the wind turbine with a stall law, this law has been selected from the experimental results, due to the high power coefficient. These results show a significant discrepancy. For a wind turbine with high-solidity The $\mathrm{Cp}$ values with variable pitch are higher between $0.3,0.4$ so it acts like a turbine unlike the $C p$ values with fixed pitch which in specific low speeds it consumes energy. In the light of this comparison, the variable pitch configuration considerably reduces the stall phenomenon, which gives it a high power coefficient. Contrary to the fixed pitch configuration which generates a lot of vortices, so it is perpetually stalled which causes its power coefficient to decrease. The classic wind turbines generally operate at a TSR 3,5, in our case for low TSR 0.4, 0.7 this wind turbine has high-solidity with fixed pitch consumes energy instead

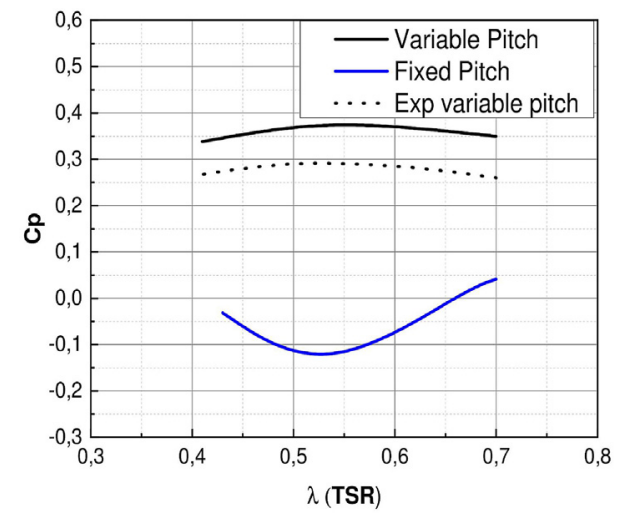

Fig. 16. Comparison of power coefficients for fixed and original variable pitch wind turbines.

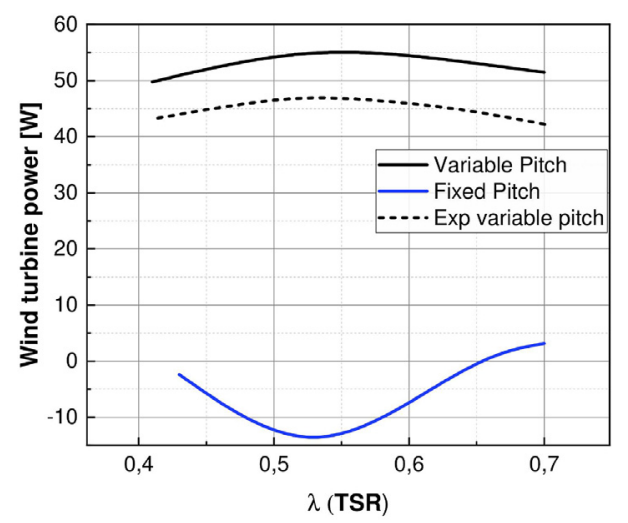

Fig. 17. Comparison of wind turbine power for fixed and original variable pitch wind turbines. of generating it up to a TSR of 0.7, however, we use the laws of not we note a considerable improvement, these laws allow this wind turbine to operate in a range of TSR which is generally dedicated to the turbines Savonius Note that with these laws these original variable pitch wind turbines surpass the savonius which gives them a considerable advantage (less noise and slower degradation) in order to locate them in urban areas. On the other hand, Fig. 16 shows that the CFD Cp values are slightly higher than the experimental data. Compared to the experience, this may be due to a negligence of the aerodynamic drag, more precisely the axis of rotation and the connecting rods, given their high-solidity. In addition, mechanical losses in general reach (30\%). This may explain this difference in the results. This power coefficient $C p$ is defined as the ratio of the obtained power to the maximum available wind power. A theoretical maximum attainable limit of $\mathrm{Cp}$ is given by the limit of Betz of $C p_{\max }=16 / 27$ which turns out to be 0.593 [35]. The same behaviour can be observed for the power curve see Fig. 17.

\subsection{The torque effect}

Torque (T) is an important parameter in the assessment of the generation capacity of a wind turbine that is not variable [36]. The ratio of torque to power coefficient in Eq. (11) is positively attached to angular velocity. The variations of the instantaneous torque of the fixed pitch wind turbine and variable pitch depending on azimuthal angle are shown in Fig. 18. The results show that during a rotation cycle the torque is practically positive for the wind turbine with variable pitch because of the particularity of this wind turbine which always faces the wind contrary to the conventional wind turbines (fixed pitch) that during a rotation cycle the torque varies between positive and negative values [37] which corresponds to the regions located in the zone contrary to the wind (from $180^{\circ}$ to $360^{\circ}$ ) stall area due to the increase in drag force. Fig. 19 shows the torque ripples for a variable blade turbine with different $T S R=0.52$ and TSR $=0.64$. Three values of the maximum torque are observed during a revolution, corresponding to the effects of the blades. Since the torque of the rotor is the sum of the torque of each blade, it can be seen when the torque of one blade reaches the maximum value while $\mathrm{T}$ of the others is close to zero. Therefore, there is a slight difference for the maximum value of this one. The regions of the rotor extreme torque occur three times during a revolution and rotor rotation is affected by inertia in these regions. The results also show that regions and maximum torque change with the TSR. In Fig. 19 it is revealed that the mean rotor results obtained with wind turbine with fixed pitch are even smaller than those obtained by

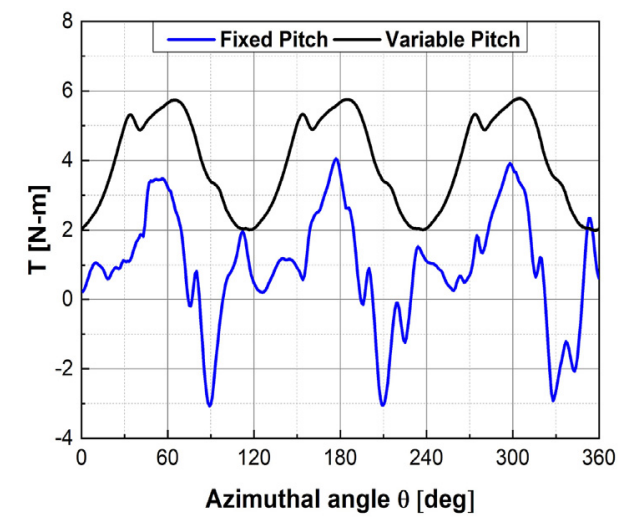

Fig. 18. Rotor torque variations during a revolution for different configurations. 


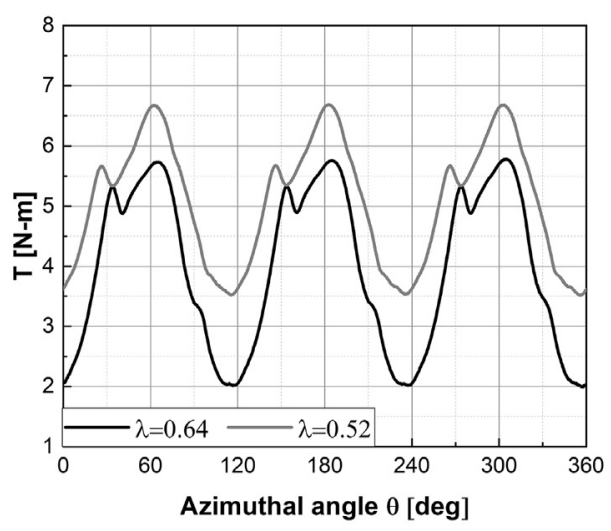

Fig. 19. Torque variations of the original variable pitch wind turbine during a turn at different TSRs.

wind turbine with variable pitch. Waves with larger TSR (TSR = $0.64)$ appear smoother than those with lower TSR $(T S R=0.52)$. In a different case from HAWT, the aerodynamic forces of VAWT change periodically with rotor rotation due to the instantaneous variation of the attack angle (AOA).

\subsection{Streamline effect}

Fig. 20 shows the 3D streamlines of the VAWT with a variable pitch simulated by the DES model at TSR $=0.52$. Since the pressure difference between the pressure side and the suction side is very large, there is an increasing pressure gradient between the two sides. It leads to the streamline of a blade that moves up and down towards the middle of the blade.

\subsection{Influence of vorticity on the wind turbine with variable and} fixed pitch

To determine some of the most important physical flow phenomena during simulations. The complex flow evolves around the blades of a vertical axis wind turbine with high-solidity and low TSR. Dynamic stall is characterized by a large recirculation zone (release of the boundary layer) that creates vortices. This can affect other rotating blades downstream. For more details on the complex flow associated with a low TSR, the vortex contours have been displayed on a plane that cuts the center through the blades of the wind turbine with a variable and fixed pitch NACA 0018, as shown in Fig. 21. In this study, the flow regimes of these VAWTs during a rotation are first characterized in three steps: attachment flux, light flux separation and massive separation (dynamic stall). The utilized

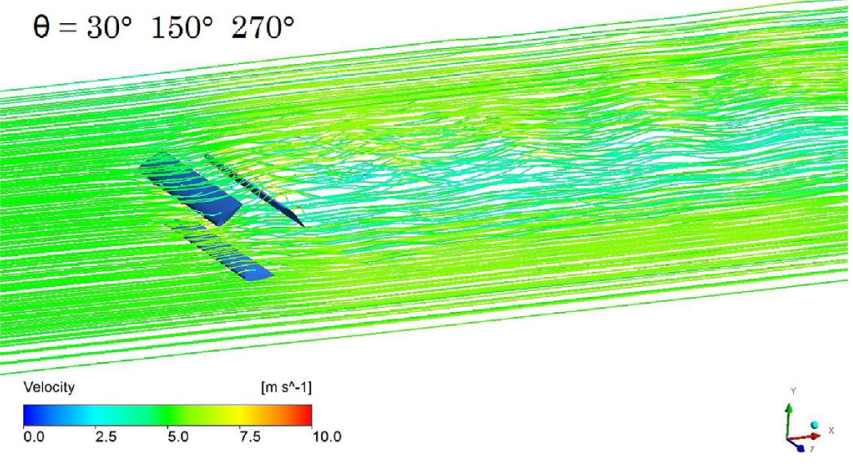

Fig. 20. Diagrams of the current line predicted by DES a TSR $=0.52$. (a)
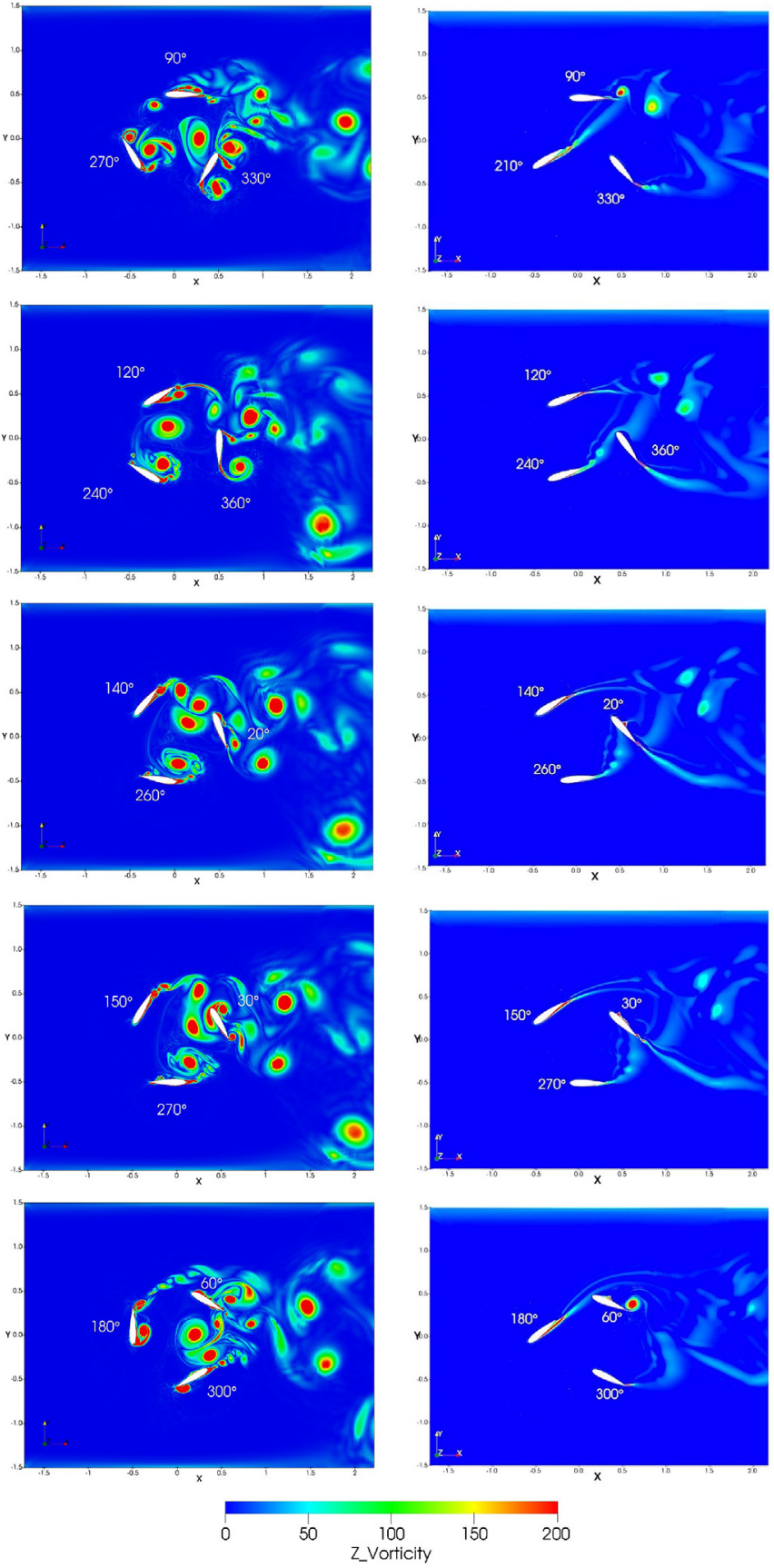

Fig. 21. Instantaneous contours of the vorticity magnitude at different azimuth angles for TSR $=0.52$ :(a):Fixed Pitch,(b):Variable Pitch.

turbulence model, i.e. DES, can solve all regions of attached flows, slightly separated flows and massively separated flows [38]. When slightly separated flows occur (Fig. 21), the stall is at the trailing edge. Fig. 21 column (a) and (b) show the vortex distribution around the rotor for a wind turbine cycle for a wind turbine with a fixed and variable pitch of TSR $=0.52$. It is shown that the wake region downstream in the variable pitch angle rotor is smaller than that of the fixed pitch angle rotor. This is related to the weaker and smaller eddies that are produced by the blades of the original variable pitch wind turbine that dissipate more quickly. In addition, in the variable pitch angle method, the current lines are attached to the surface of the NACA0018 blades, while a separation is detected 
for the blades of the fixed pitch wind turbine. This justifies the decreasing torque coefficient in Fig. 22. In addition, the pressure gradient on the leading edge of the turbine in the variable pitch turbine is wider than that of the fixed-pitch turbine, resulting in higher torque production [39]. It can be seen that for a fixed pitch wind turbine, the rotor blade develops maximum lift. In Fig. 21 column (a), it is observed that the final vortex is at its maximum force in the case for the $90^{\circ}$ rotor blade. The intensity of the vortex and its extent are maximum for this blade in this (phase) Fig. 21 column (a). When this rotor blade reaches the rotor location at $30^{\circ}$ for a fixed-pitch wind turbine, the vortex range of the rotor end decreases, while the vortex amplitude of the rotor end at $30^{\circ}$ decreases Fig. 21 column (a). By the time the rotor reaches the blade location at $150^{\circ}$, it appears to be at a minimum. This force change is due to the variable developed blade lift of the turbine rotor when it rotates at different phase angles. The wake caused by the peak vortex extends over a considerable proportion of the area swept by the rotor blade Fig. 21 column (a). As the wake segment continues to flow downstream, it interacts with the blades behind. This will result in changes in the distribution of the surface pressure of this blade, but can also change the transition points from laminar to turbulent flow in the turbulent flow in the rotor blade. This is due to the high levels of turbulence in the wake and the relatively small number of Reynolds flow on this rotor. Unlike the original variable pitch wind turbine Fig. 21 column (b), which has the particularity of always facing the wind. In Fig. 21 column (b), two eddies appear towards the trailing edge. A series of different vortex amplitudes around the aerodynamic profile is observed. When the blade rotates towards the azimuth of $30^{\circ}$ Fig. 21 column (b), the dynamic stall develops further and the vortex derived from the leading edge moves towards the center of the wing until it reaches the trailing edge at $60^{\circ}$. Meanwhile, an inverted wormhole at the trailing edge is again induced. As can be seen, the expected results show a large vortex detached from the suction surfaces of the blades. Unlike a conventional VAWT in perpetual stall. The variable-pitch mechanism reduces the angle of attack and a smaller vortex is formed in the downstream region. In other words, in fixed-pitch wind turbines with greater solidity (as shown in Figs. 21 and 23), the chances of encountering vortices detached from the upstream to the downstream part are increased. As a result, the lift force and output power coefficient were reduced. In contrast, in a VAWT with variable pitch (Fig. 21 column (b)), flow separation, vortex interaction and dynamic stall oscillation were reduced. It can be predicted that, in variable-pitch wind turbines, the vibrations and fatigue stresses acting on the rotor and structure will be reduced. The reason for the increase in turbine power in high-pitch cases can be explained by the fact that using the variable-pitch angle technique reduces the chance of encountering high-solidity, upstream-
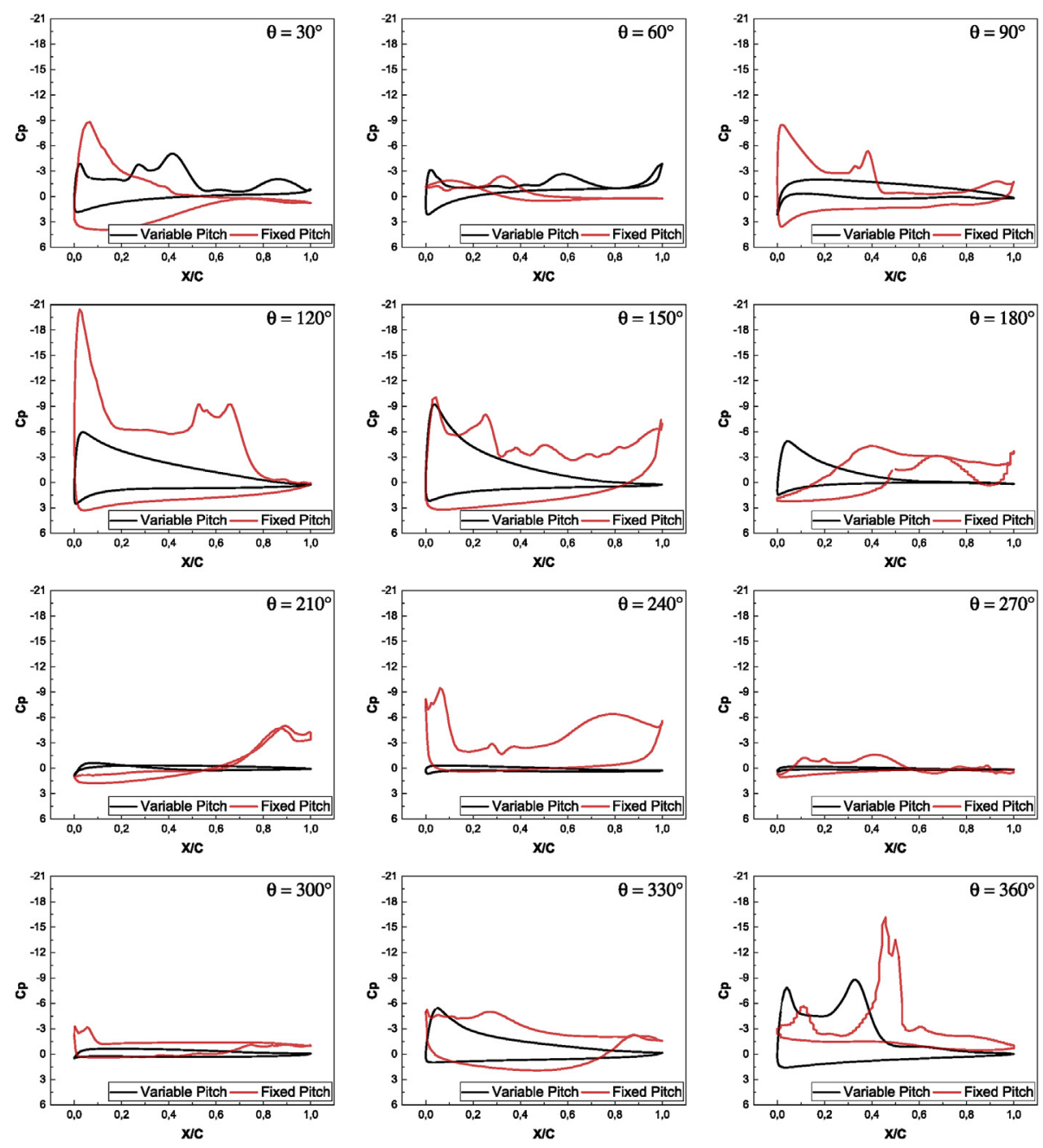

Fig. 22. Pressure coefficient curves of the central section of the wind turbine during one turn. 
to-downstream, lifted eddies, thereby reducing flow separation and drag force on the blades. The LES, which is engaged in the DES model outside the boundary zone, is able to capture the vortex more efficiently on a large scale and obtain more accurate information on the flow field.

\subsection{Drag force effect}

In aerodynamics, the drag corresponds to the air resistance, which increases with the surface exposed to the direction of air flow. The drag is a force that acts on the wind turbines. This force tends to counter the forward movement of the blade and should therefore be as low as possible. The drag is calculated as follows:

$F_{d}=\frac{1}{2} \rho S C_{d} V^{2}$

In Fig. 24 we can see that for a rotation cycle the drag of the fixed pitch wind turbine is larger than that of the original variable pitch wind turbine. There are three azimuthal angle phases where the drag is at most, while in Fig. 24 the results show a comparison between the different wind turbine configurations for several specific TSR speeds, the result is that the fixed pitch wind turbine has a high drag force, which is bad, unlike the configuration of the original variable pitch wind turbine. As a result, with this variable pitch configuration we will have a high power coefficient with low specific speeds (TSR) and this will preserve the wind turbine.

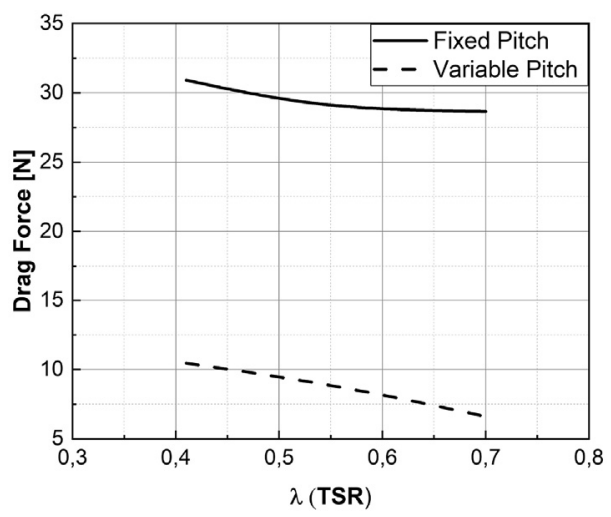

Fig. 23. The drag force of the different wind turbine configurations depends on the specific speed TSR.

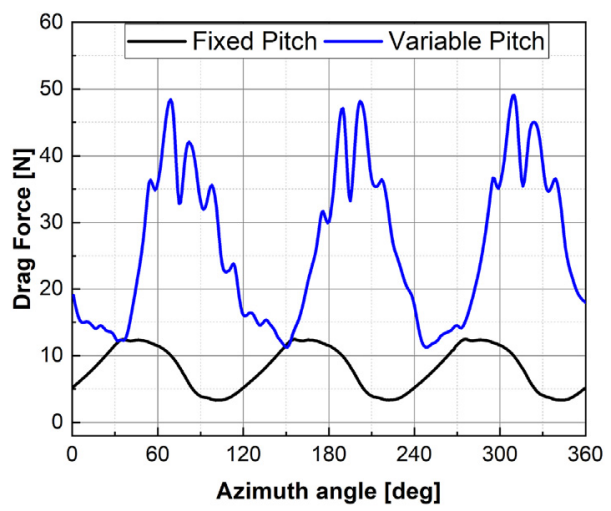

Fig. 24. The power of dragging a fixed and original variable pitch wind turbine for one revolution.

\section{Conclusion}

This paper studies an original variable-pitch VAWT in the case of high-solidity rotor with straight-blades and which can be useful for implementation in urban areas. Experimental investigations in wind tunnel and also numerical simulations have been successfully conducted. The flow simulation was performed using DES turbulence model and sliding mesh method. The simulation of blade pitch variation during the rotor rotation was made possible using a specific user defined function UDF. The simulations permitted to obtain the wind turbine aerodynamic performances represented by the power coefficient $\mathrm{Cp}$ depending on tip speed ratio TSR. The simulations were firstly conducted in case of the variable pitch VAWT which was investigated in wind tunnel. Then another simulation was conducted in the case of the same rotor with fixed pitch. Thus, it was possible to study the effect of pitch variation on the aerodynamic performances. The analysis of impact obtained parameters is essential to improve the functionality of vertical axis wind turbines. The main conclusions of the study can be summarized as follows:

(1) The studied variable pitch VAWT has major advantage respectively to fixed pitch VAWT in the case of high-solidity rotor where the blade wake is large. As the blade pitch is adapted to flow, then the shedding vortices are much lower. In this case the downstream blade operates better and produces more useful torque. The presented CFD results show that variable pitch VAWT has significantly higher power than fixed pitch VAWT.

(2) Pitch variation of the blade reduces flow separation and as result the drag forces are lower. As consequence, structure vibrations and fatigue stresses acting on the rotor and the shaft are also reduced.

(3) The variation of blade pitch leads to narrower wake with smaller vortices which dissipate rapidly, especially for this type of high-solidity wind turbine. As consequence, the dynamic forces applied on the downstream blade due to this wake are lower.

(4) Our numerical simulations represent well the aerodynamic performances of the wind turbine and flow through the wind turbine rotor. However, some discrepancy is observed due to the phenomenon of dynamic stall and blade vortex interaction. The improvement of this discrepancy requires a thorough study.

\section{Acknowledgments}

I would like to thank Pr. Massouh, Dr. Dobrev for welcoming me to their team. I also thank him for welcoming me to Aerodynamics laboratory at Arts et Métiers Paris Tech.

\section{References}

[1] Cai X, Gu R, Pan P, Zhu J. Unsteady aerodynamics simulation of a full-scale horizontal axis wind turbine using CFD methodology. Energy Convers Manag 2016;112:146-56.

[2] Mertens S. Wind energy in the built environment: concentrator effects of buildings. 2006.

[3] Stankovic S, Campbell N, Harries A. Urban wind energy. Routledge; 2009.

[4] D. G. J. Marie, Turbine having its rotating shaft transverse to the flow of the current, 1931. US Patent 1,835,018.

[5] Zamani M, Maghrebi MJ, Varedi SR. Starting torque improvement using jshaped straight-bladed darrieus vertical axis wind turbine by means of numerical simulation. Renew Energy 2016;95:109-26.

[6] Madsen HA, Lundgren K. The Voith-Schneider wind turbine: some theoretical and experimental results on the aerodynamics of the Voith-Schneider wind turbine, Institute of Industrial Constructions and energy technology. Aalborg University Centre; 1980. 
[7] G. M. Tierney, Cycloidal vtol uav, 2005. US Patent 6,932,296.

8] Xisto CM, Páscoa JC, Leger JA, Trancossi M. Wind energy production using an optimized variable pitch vertical axis rotor. 2014. ASME Paper No. IMECE2014-38966.

[9] Sagharichi A, Maghrebi MJ, ArabGolarcheh A. Variable pitch blades: an approach for improving performance of Darrieus wind turbine. J Renew Sustain Energy 2016;8:053305.

[10] A. Curutchet, S. Grosmangin, R. Fourton, G. Corde, Rotary machine comprising a rotor placed in a fluid and equipped with orientable blades, 2017. US Patent $9,841,003$.

[11] Duquette MM, Visser KD. Numerical implications of solidity and blade number on rotor performance of horizontal-axis wind turbines. Trans Am Soc Mech Eng J Solar Energy Eng 2003;125:425-32.

[12] Eriksson S, Bernhoff H, Leijon M. Evaluation of different turbine concepts for wind power. Renew Sustain Energy Rev 2008;12:1419-34.

[13] Paraschivoiu I, Trifu O, Saeed F. H-darrieus wind turbine with blade pitch control. Int J Rotating Mach 2009;2009.

[14] Bianchini A, Ferrara G, Ferrari L. Pitch optimization in small-size darrieus wind turbines. Energy Procedia 2015;81:122-32.

[15] Li C, Xiao Y, Xu Y-l, Peng Y-x, Hu G, Zhu S. Optimization of blade pitch in hrotor vertical axis wind turbines through computational fluid dynamics simulations. Appl Energy 2018;212:1107-25.

[16] Rezaeiha A, Kalkman I, Blocken B. Effect of pitch angle on power performance and aerodynamics of a vertical axis wind turbine. Appl Energy 2017;197: 132-50.

[17] Jain P, Abhishek A. Performance prediction and fundamental understanding of small scale vertical axis wind turbine with variable amplitude blade pitching. Renew Energy 2016;97:97-113.

[18] Abdalrahman G, Melek W, Lien F-S. Pitch angle control for a small-scale darrieus vertical axis wind turbine with straight blades (h-type vawt). Renew Energy 2017;114:1353-62.

[19] Xisto CM, Páscoa JC, Trancossi M. Geometrical parameters influencing the aerodynamic efficiency of a small-scale self-pitch high-solidity VAWT. J Sol Energy Eng 2016;138:031006.

[20] Kavade RK, Ghanegaonkar PM. Performance evaluation of small-scale vertical axis wind turbine by optimized best position blade pitching at different tip speed ratios. J Inst Eng 2018:1-10. Series C.

[21] Shahizare B, Nik-Ghazali N, Chong WT, Tabatabaeikia S, Izadyar N, Esmaeilzadeh A. Novel investigation of the different Omni-direction-guidevane angles effects on the urban vertical axis wind turbine output power via three-dimensional numerical simulation. Energy Convers Manag 2016;117:206-17.

[22] Gharali K, Johnson DA. Dynamic stall simulation of a pitching airfoil under unsteady freestream velocity. J Fluids Struct 2013;42:228-44.
[23] Almohammadi KM, Ingham DB, Ma L, Pourkashanian M. Modeling dynamic stall of a straight blade vertical axis wind turbine. J Fluids Struct 2015;57: 144-58.

[24] Lei H, Su J, Bao Y, Chen Y, Han Z, Zhou D. Investigation of wake characteristics for the offshore floating vertical axis wind turbines in pitch and surge motions of platforms. Energy 2019;166:471-89.

[25] Lee Y-T, Lim H-C. Numerical study of the aerodynamic performance of a 500 W Darrieus-type vertical-axis wind turbine. Renew Energy 2015;83:407-15.

[26] Roh S-C, Kang S-H. Effects of a blade profile, the Reynolds number, and the solidity on the performance of a straight bladed vertical axis wind turbine. J Mech Sci Technol 2013;27:3299-307.

[27] Kirke BK, Paillard B. Predicted and measured performance of a vertical axis wind turbine with passive variable pitch compared to fixed pitch. Wind Eng 2017:41:74-90.

[28] Sheldahl RE, Klimas PC. Aerodynamic characteristics of seven symmetrical airfoil sections through 180-degree angle of attack for use in aerodynamic analysis of vertical axis wind turbines. Albuquerque, NM (USA): Sandia National Labs.; 1981. Technical Report.

[29] Elkhoury M, Kiwata T, Aoun E. Experimental and numerical investigation of a three-dimensional vertical-axis wind turbine with variable-pitch. J Wind Eng Ind Aerodyn 2015;139:111-23.

[30] Larose GL, D'Auteuil A. Effect of local air compressibility on the aerodynamics of rectangular prisms at Mach number below 0.3. In: 6th international colloquium on bluff body aerodynamics from, vol. 7; 2008. p. 20.

[31] Fluent A. Ansys fluent udf manual. USA: ANSYS Inc.; 2015.

[32] Zhang LX, Liang YB, Liu XH, Jiao QF, Guo J. Aerodynamic performance prediction of straight-bladed vertical axis wind turbine based on CFD. Adv Mech Eng 2013;5:905379.

[33] Spalart PR, Jou WH, Strelets M, Allmaras SR. Comments on the feasibility of LES for wings, and on a hybrid RANS/LES approach. Advances in DNS/LES 1997:1:4-8.

[34] Hansen MO. Aerodynamics of wind turbines. Routledge; 2015.

[35] Paraschivoiu I. Wind turbine design: with emphasis on Darrieus concept. Presses inter Polytechnique; 2002.

[36] Balduzzi F, Bianchini A, Maleci R, Ferrara G, Ferrari L. Critical issues in the CFD simulation of Darrieus wind turbines. Renew Energy 2016;85:419-35.

37] Lei H, Zhou D, Bao Y, Li Y, Han Z. Three-dimensional improved delayed detached eddy simulation of a two-bladed vertical axis wind turbine. Energy Convers Manag 2017;133:235-48.

[38] Shur ML, Spalart PR, Strelets MK, Travin AK. A hybrid RANS-LES approach with delayed-DES and wall-modelled LES capabilities. Int J Heat Fluid Flow 2008;29:1638-49.

[39] Burton T, Sharpe D, Jenkins N, Bossanyi E. Wind energy handbook. John Wiley \& Sons: 2001. 